\title{
Trombosis séptica portal secundaria a diverticulitis
}

\section{Portal septic thrombophlebitis secondary to diverticulitis}

Sebastián Guerra-Zarama ${ }^{1 凶}$, Cristian Iván García-Rincón ${ }^{2 \bowtie}$, Javier Mauricio González-Pérez ${ }^{3 凶}$, Gustavo Roncancio-Villamil ${ }^{4 凶}$

${ }^{1}$ Residente de Medicina Interna, Universidad CES, Medellín.

2 Especialista en enfermedades infecciosas, Clínica Universitaria Bolivariana, Clínica Cardio VID, Medellín. Docente Escuela de Ciencias de la salud - Universidad Pontificia Bolivariana.

${ }^{3}$ Especialista en enfermedades infecciosas, Hospital Manuel Uribe Ángel, Envigado.

${ }^{4}$ Especialista en enfermedades infecciosas, Clínica Cardio VID, Medellín. Docente Escuela de Ciencias de la salud - Universidad Pontificia Bolivariana.

Fecha correspondencia:

Recibido: octubre 20 de 2020.

Revisado: abril 28 de 2021.

Aceptado: mayo 21 de 2021.

Forma de citar:

Guerra-Zarama S, García-Rincón

$\mathrm{Cl}$, González-Pérez JM,

Roncancio-Villamil G. Trombosis

séptica portal secundario a

diverticulitis. Rev CES Med. 2021;

35(2): 135-145. 10.21615/

cesmedicina. 5935

\section{Open access}

\section{Derecho de autor}

Licencia creative commons

Ética de publicaciones

Revisión por pares

Gestión por Open Journal System

DOI: http://dx.doi.org/10.21615/

cesmedicina.5935

ISSNe 2215-9177

ISSN 0120-8705

Publica con nosotros

\section{Resumen}

La trombosis séptica de la vena porta es una complicación infrecuente de la diverticulitis, la apendicitis y de otras infecciones intraabdominales. Se asocia a elevadas tasas de complicaciones, como isquemia mesentérica, absceso hepático, hipertensión portal o muerte. Se presenta el caso de un paciente de 67 años quien consultó por fiebre, dolor abdominal e ictericia, y en quien se documentó bacteriemia por Escherichia coli y Streptococcus dysgalactiae. En las imágenes se identificó trombosis de la porta hepática secundaria a enfermedad diverticular aguda, lo que configura un diagnóstico de pileflebitis séptica. El paciente recibió terapia antibiótica dirigida, sin anticoagulación, obteniéndose adecuada respuesta clínica. Se realiza una discusión sobre el diagnóstico, etiología y tratamiento de la trombosis séptica portal.

Palabras clave: Colangitis; Diverticulitis; Vena porta; Trombosis séptica portal. 


\section{Abstract}

Septic thrombosis of the portal vein is a rare complication of diverticulitis, appendicitis and other intra-abdominal infections; it is associated with high rates of complications, such as mesenteric ischemia, liver abscess, portal hypertension or death. We present the case of a 67-year-old patient who consulted for fever, abdominal pain and jaundice, and in whom Escherichia coli and Streptococcus dysgalactiae bacteremia was documented; images showed hepatic portal thrombosis secondary to acute diverticular disease, which configures a diagnosis of septic pylephlebitis. The patient received targeted antibiotic therapy without anticoagulation, with adequate clinical response. The diagnosis, etiology, and treatment of portal septic thrombosis are discussed.

Keywords: Cholangitis; Diverticulitis; Portal vein; Portal septic thrombosis.

\section{Introducción}

La tromboflebitis séptica portal o pileflebitis es la trombosis de la vena porta o de sus ramas, de manera concomitante o posterior a una infección bacteriana del tracto gastrointestinal o a procesos inflamatorios intraabdominal ${ }^{(1)}$. Es una complicación rara, y por lo tanto es difícil establecer su incidencia, pero se ha calculado que puede llegar a afectar a 2,7 por cada 100000 personas, con notable mortalidad (11 a $32 \%$ ) cuando el diagnóstico o tratamiento no son adecuados $^{(2-4)}$. La principal causa de tromboflebitis séptica portal es la diverticulitis, y entre sus principales complicaciones se encuentran absceso hepático, isquemia intestinal, hipertensión portal y muerte $e^{(4,5)}$.

Se presenta el caso de un paciente quien desarrolló tromboflebitis séptica portal secundaria a diverticulitis, discutiendo el impacto de la anticoagulación, tipo y tiempo de terapia antimicrobiana; además de la necesidad de exploraciones adicionales. Se revisa la literatura actual sobre el tema.

\section{Presentación del caso}

Un hombre de 67 años con antecedentes de hipertensión arterial y enfermedad pulmonar obstructiva crónica consultó en el servicio de urgencias por dos semanas de diarrea, dolor abdominal generalizado y fiebre. Al ingreso estaba ictérico, con tensión arterial de $110 / 60 \mathrm{~mm} \mathrm{Hg}$, frecuencia cardíaca de $110 \mathrm{ppm}$, respiratoria de $26 \mathrm{rpm}$, temperatura de $38,6^{\circ} \mathrm{C}$, 
y dolor a la palpación profunda en hemiabdomen derecho, con signo de Murphy positivo. Ante la presencia de dolor, fiebre e ictericia (el paciente negaba acolia u orina hiperpigmentada) se sospechó colangitis y se inició tratamiento con ceftriaxona, previa toma de hemocultivos (cuadro 1).

Cuadro 1. Paraclínicos al ingreso

\begin{tabular}{ll}
\hline \multicolumn{1}{c}{ Paraclínico } & \multicolumn{1}{c}{ Resultado } \\
\hline $\mathrm{pH}$ & 7,51 \\
$\mathrm{pCO}_{2}$ & $25,6 \mathrm{~mm} \mathrm{Hg}$ \\
$\mathrm{HCO}_{3}$ & $20,4 \mathrm{mmol} / \mathrm{L}$ \\
$\mathrm{BE}$ & $-0,7 \mathrm{mmol} / \mathrm{L}$ \\
$\mathrm{INR}$ & 1,37 \\
$\mathrm{TPT}$ & $42,6 \mathrm{segundos}$ \\
$\mathrm{AST}$ & $48 \mathrm{U} / \mathrm{L}$ \\
$\mathrm{ALT}$ & $44 \mathrm{U} / \mathrm{L}$ \\
Fosfatasa alcalina & $148 \mathrm{U} / \mathrm{L}$ \\
Bilirrubina total & $1,05 \mathrm{mg} / \mathrm{dL}$ \\
Bilirrubina indirecta & $0,38 \mathrm{mg} / \mathrm{dL}$ \\
BUN & $19,7 \mathrm{mg} / \mathrm{dL}$ \\
PCR & $7,9 \mathrm{mg} / \mathrm{dl}$ \\
Hb & $9,1 \mathrm{~g} / \mathrm{dL}$ \\
Leucocitos & $22.384 \times 10^{6} / \mu \mathrm{L}$ \\
Neutrófilos & $96,1 \%$ \\
Plaquetas & $103000 \times \mathrm{mm}^{3}$ \\
\hline
\end{tabular}

Una ecografía hepatobiliar reportó hígado con parénquima heterogéneo irregular, con aumento de la ecogenicidad parietal de la vía biliar intrahepática; además de imagen nodular de $4 \times 1,5 \mathrm{~cm}$ y paredes gruesas con respuesta al Doppler. Ante el hallazgo ecográfico se consideraron como posibilidades diagnósticas: colangitis en fase inicial, lesión intraductal intrahepática sobre-infectada, hamartoma biliar sobre-infectado y trombosis del sistema porta intrahepático (figura 1). Después de 48 horas el paciente seguía febril, con ascenso de las bilirrubinas y de la proteína $C$ reactiva. 
Los hemocultivos resultaron ser positivos para Escherichia coli resistente a ampicilina y Streptococcus dysgalactiae. Se decidió ajustar el antibiótico a piperacilina/tazobactam y realizar tomografía de abdomen simple y contrastada que evidenció hígado con parénquima hipodenso y ausencia de llenado parcial en territorio de la porta hepática y su rama izquierda por trombos (figura 2). Además, en las asas intestinales se observaba inflamación en la válvula ileocecal y múltiples divertículos en todo el colon; configurando un diagnóstico de diverticulitis aguda y trombosis séptica portal asociada, también conocida como pileflebitis.

Después de iniciar piperacilina/tazobactam el paciente presentó defervescencia, mejoría clínica y depuración de bacteriemia, se decidió que ante el riesgo de sangrado no se beneficiaba dar anticoagulación. Adicionalmente, una colonoscopia documentó enfermedad diverticular pancolónica y después de 21 días de tratamiento presentó resolución completa del cuadro clínico. Luego de un año de seguimiento el paciente persiste asintomático.

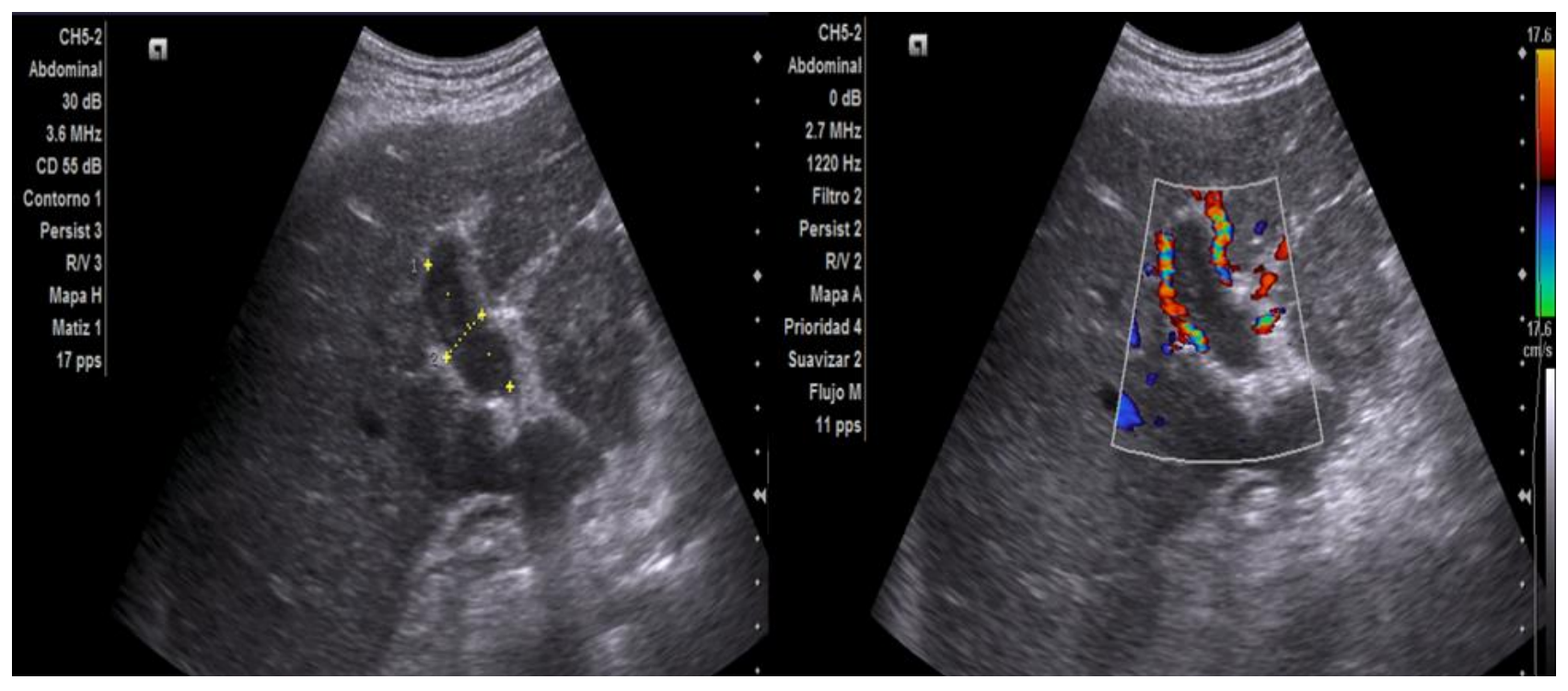

Figura 1. Ecografía de abdomen.

Se observa hígado con parénquima heterogéneo irregular, con aumento de la ecogenicidad parietal de la vía biliar intrahepática, además de imagen nodular de $4 \times 1,5 \mathrm{~cm}$ y paredes con respuesta a la señal

Doppler. 


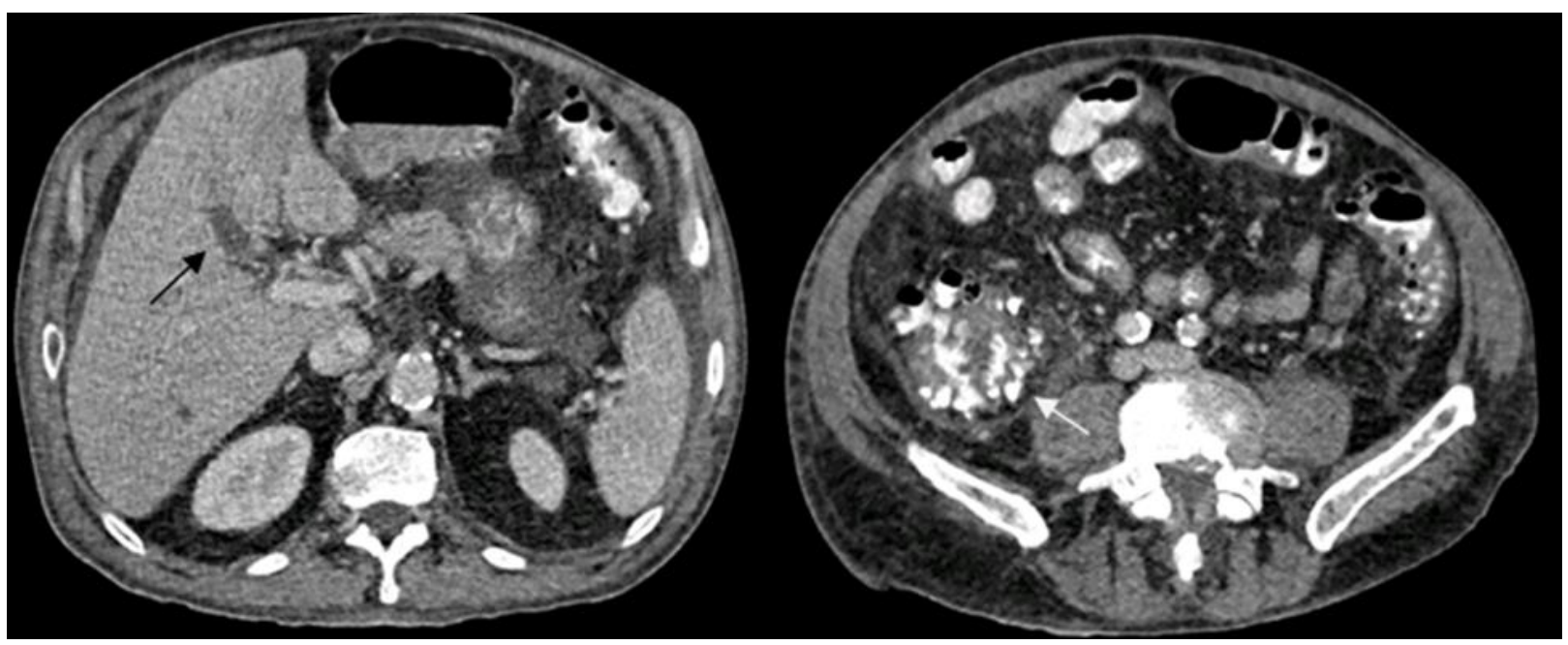

Figura 2. Tomografía contrastada de abdomen.

Se observa hígado de parénquima hipodenso y ausencia de llenado parcial en territorio de la porta hepática y su rama izquierda por trombos (flecha negra). Además, en las asas intestinales, se observó inflamación en la válvula ileocecal (flecha blanca) y múltiples divertículos en todo el colon.

\section{Discusión}

La tromboflebitis séptica portal es una condición cuya primera descripción se remonta a 1846 cuando Waller la descubre, en necropsias, como fuente de abscesos hepáticos ${ }^{(6)}$. También se le ha denominado pileflebitis. Típicamente, hay trombosis de la vena porta o de sus ramas, después o simultáneamente a una infección bacteriana del tracto gastrointestinal o de procesos inflamatorios intraabdominales (enfermedad de Behçet, enfermedad inflamatoria intestinal, neoplasia, entre otras) $)^{(1)}$.

La principal causa de la tromboflebitis séptica portal es la diverticulitis, como en el caso descrito. Llama la atención que el paciente no presentó evidencia clínica de diverticulitis y su diagnóstico se estableció como parte del estudio etiológico de la tromboflebitis séptica portal. Se sabe que la tromboflebitis séptica portal inicia cuando el proceso inflamatorio piógeno se extiende hasta el drenaje periférico de las venas mesentéricas. Allí se produce activación de la cascada inflamatoria a través de la interacción entre el agente infeccioso y el endotelio, lo que precipita la trombosis séptica, con posterior embolia y compromiso de la vena porta ${ }^{(5)}$. 
Otras causas asociadas incluyen apendicitis, pancreatitis y raramente enfermedad intestinal inflamatoria y colecistitis ${ }^{(7)}$. Plemmons et al. encuentran que en el $32 \%$ de los casos no hay etiología identificable ${ }^{(8)}$. En la serie de Choudhry et al. se identifica a la pancreatitis como la primera causa de tromboflebitis séptica portal, aunque este estudio fue realizado en un centro de referencia para pacientes con pancreatitis ${ }^{(9)}$. El cuadro 2 resume las condiciones reportadas $y$ asociadas con la enfermedad ${ }^{(2,3,10,11)}$.

Cuadro 2. Condiciones asociadas al desarrollo de tromboflebitis séptica portal

\begin{tabular}{|l|}
\hline Cirugía abdominal reciente \\
\hline Cirugía abdominal remota* \\
\hline Inmunosupresión \\
\hline Pancreatitis crónica \\
\hline Tumores sólidos \\
\hline Neoplasias hematológicas \\
\hline Trastornos de la coagulación \\
\hline Tabaquismo \\
\hline Cirrosis \\
\hline Hipertensión portal \\
\hline Uso anticonceptivos orales \\
\hline
\end{tabular}

*Se define como aquella que sucedió hace seis meses o más

El diagnóstico requiere un alto grado de sospecha, pues sus manifestaciones clínicas son inespecíficas, siendo las más comunes fiebre y dolor abdominal. Otros hallazgos como náuseas, vómito, hepatomegalia e ictericia son menos frecuentes ${ }^{(10)}$. Plessier et al. reportan ascitis en el $50 \%$ de los pacientes, en la mayoría por imágenes ${ }^{(12)}$. No hay síntomas o signos patognomónicos, pero debe tenerse siempre en cuenta como diagnóstico diferencial de colangitis, abscesos hepáticos y pancreatitis; especialmente cuando hay fiebre persistente asociada a dolor abdominal, alteración del perfil bioquímico hepático y bacteriemia en ausencia de enfermedad de la vía biliar, como el caso descrito ${ }^{(13)}$.

La mayoría de los pacientes cursan con leucocitosis, bandemia, elevación de la fosfatasa alcalina (3-4 veces de su valor normal) y de la gamma-glutamil transferasa (5-10 veces su valor). En contraste con lo reportado, la elevación de bilirrubinas en suero es rara, así como la presencia de ictericia ${ }^{(10)}$. 
Entre las ayudas por imagen se cuenta con el Doppler hepático, la resonancia magnética y la tomografía abdominal contrastada. El Doppler, por ser operador dependiente y por la cantidad de aire en colon, puede dar falsos negativos; mientras que la resonancia tiene como principales inconvenientes sus costos y poca disponibilidad. La tomografía abdominal con contraste oral y venoso se prefiere como primera opción por su capacidad para identificar la trombosis y el posible origen de esta ${ }^{(10)}$.

Ningún hallazgo en la tomografía debe considerarse patognomónico; la asociación de imágenes y las manifestaciones clínicas ayudan a establecer el diagnóstico. Se han descrito que facilitan el diagnóstico el descubrimiento de una fuente infecciosa (diverticulitis, apendicitis, entre otras), asociado a compromiso de venas mesentéricas, vena porta o sus ramas intrahepáticas ya sea por trombos o aire endovascular, siendo este último un hallazgo temprano y, finalmente, anormalidades intrahepáticas como realce heterogéneo y abscesos ${ }^{(1,5)}$.

Siempre deben solicitarse hemocultivos, pues su rendimiento es cercano al $80 \%$, encontrando compromiso polimicrobiano hasta en la mitad de los $\operatorname{casos}^{(1,9,11)}$. Hay discrepancias sobre el principal microorganismo causal, aunque hay acuerdo en que los más comunes son Streptococcus del grupo viridans, Escherichia coli, Bacteroides fragilis, Proteus mirabilis y Enterobacter $s p p^{(11,14)}$. En una serie de casos en Taiwán se reporta a Klebsiella pneumoniae como patógeno emergente ${ }^{(15)}$. En el paciente reportado se demostró bacteriemia mixta por $E$. coli y $S$. dysgalactiae, resaltando la importancia de buscar esta complicación cuando se aísla más de un enteropatógeno en hemocultivos.

Al respecto del tratamiento, está sustentado principalmente en series de casos. Un tratamiento empírico debería dar cubrimiento para enterobacterias y anaerobios y ajustarse según la sensibilidad del antibiograma. El paciente fue tratado por 21 días con antibiótico intravenoso, obteniendo mejoría completa y sin recaídas en cuatro semanas y después de un año de seguimiento. Algunos autores coinciden en administrar siete a 14 días de antibiótico endovenoso y posteriormente continuar por dos a cuatro semanas con el tratamiento por vía oral $^{(10,14,16)}$. El manejo de esta enfermedad es eminentemente médico y no requiere intervención quirúrgica ni procedimientos endovasculares ${ }^{(9,11)}$. Sin embargo, ante la presencia de una enfermedad que requiera intervención quirúrgica inmediata, tal como colangitis o diverticulitis perforada, no se debe retrasar el manejo operatorio de la causa de base e ir de la mano con el manejo médico, para tener un control adecuado del foco infeccioso ${ }^{(9)}$. 
Mayo - agosto de 2021

No hay consenso sobre la necesidad de anticoagulación sistémica de la tromboflebitis séptica portal. Plemmons et al. describen que quienes recibieron anticoagulación pasaron de tener una sobrevida del 60 al $100 \%$, mientras que Kanellopoulou et al. describen que el uso de anticoagulación temprana se relaciona con menos secuelas y recanalización más rápida de la circulación portal, hallazgos que más reciente también encuentran Choundry et al. ${ }^{(8,9,11,17)}$. Algunos estudios trasversales con pocos pacientes, no han logrado establecer beneficio entre administrar o no terapia anticoagulante en la mayoría de los pacientes ${ }^{(18)}$.

Hay acuerdo general en el uso de anticoagulación sistémica solo cuando hay hipercoagulabilidad sistémica demostrada como causa de la tromboflebitis o cuando se demuestra infección por Bacteroides spp. En relación con la infección por Bacteroides spp. se ha encontrado que esta familia de microorganismos conlleva aumento en la producción transitoria de anticardiolipinas, favorece la agregación de fibrina y degradación de heparina ${ }^{(10,11,14)}$.

Otro punto que sí es más claro es que la búsqueda activa de trombofilias no aporta al pronóstico ni tratamiento, siendo quizás la mejor evidencia disponible al momento una cohorte de 67 pacientes que se siguió por 19 años y de los cuales a 49 se les realizó búsqueda de trombofilias, siendo todos negativos ${ }^{(17)}$.

La Asociación Europea para el Estudio del Hígado (EASL, por sus siglas en inglés) incluye el uso de anticoagulación por seis meses aludiendo a reportes de bajas tasas de sangrado y mejores desenlaces ${ }^{(13)}$; sin embargo, esta es una referencia cuyo manejo está centrado en trombosis de otras causas, sin que haya un comentario particular sobre la tromboflebitis de etiología infecciosa, la cual se asume como un fenómeno transitorio y autoreversible. El Colegio Americano de Médicos del Tórax considera que se debe anticoagular si la trombosis es sintomática y no recomiendan tratamiento cuando es un hallazgo incidental ${ }^{(19)}$.

Luego de analizar al paciente y sus particularidades se decidió no dar tratamiento anticoagulante, dado que presentaba una enfermedad diverticular severa, que tiene tasas de sangrado de hasta 30 \% y que podrían aumentar si de manera concomitante hay hipertensión arterial $^{(20)}$. 
La tromboflebitis séptica portal es una enfermedad que si no es tratada de manera rápida y agresiva puede terminar en distintas complicaciones como isquemia intestinal, absceso hepático, hipertensión portal crónica o muerte ${ }^{(4)}$. La mayoría de abscesos hepáticos se limitan al lóbulo izquierdo, debido a que flujo portal laminar favorece el crecimiento bacteriano a diferencia del lóbulo derecho que posee un flujo irregular ${ }^{(21)}$.

\section{Conclusiones}

La tromboflebitis séptica portal es una complicación rara de múltiples enfermedades intraabdominales y debería considerarse al evaluar a un paciente con fiebre, dolor abdominal y bacteriemia, en especial cuando es mixta. Gracias al avance en imágenes diagnósticas puede hacerse un diagnóstico precoz y proporcionar un tratamiento adecuado, el cual se realiza con antibióticos de manera prolongada y solo hay indicaciones formales de anticoagulación si hay hipercoagulabilidad o infección demostrada por Bacteroides spp. Se requiere evidencia clínica de buena calidad para determinar el tiempo adecuado de antibioticoterapia, las indicaciones específicas de anticoagulación y el pronóstico real de la enfermedad.

\section{Aspectos éticos}

Este es un reporte descriptivo, sin riesgo para el paciente. El paciente firmó el consentimiento informado para uso de sus datos y se discutió la pertinencia del reporte en el comité de ética de la institución.

\section{Agradecimientos}

A la Clínica CardioVID por permitir el uso de la información del paciente. Los autores no declaran conflictos de interés.

\section{Bibliografía}

1. Ames JT, Federle MP. Septic Thrombophlebitis of the Portal Venous System: Clinical and Imaging Findings in Thirty-Three Patients. Dig Dis Sci. 2011;56(7):2179-84.

2. Abraham MN, Mathiason MA, Kallies KJ, Cogbill TH, Shapiro SB. Portomesenteric venous thrombosis: a community hospital experience with 103 consecutive patients. Am J Surg. 2011;202(6):759-64.

3. Acosta S, Alhadad A, Svensson P, Ekberg O. Epidemiology, risk and prognostic factors in mesenteric venous thrombosis. Br J Surg. 2008;95(10):1245-51. 
4. Ufuk F, Herek D, Karabulut N. Pylephlebitis Complicating Acute Appendicitis: Prompt Diagnosis with Contrast-Enhanced Computed Tomography. J Emerg Med. 2016;50(3):e147-9.

5. Balthazar EJ, Gollapudi P. Septic thrombophlebitis of the mesenteric and portal veins: CT imaging. J Comput Assist Tomogr. 2000; 24:755-760.

6. Bolt RJ. Diseases of the hepatic blood vessels. In: Bockus HL, ed. Gastroenterolog)r. 4th ed. Philadelphia: WB Saunders, 19853259- 3277.

7. Chirinos JA, Garcia J, Alcaide ML, Toledo G, Baracco GJ, Lichtstein DM. Septic Thrombophlebitis: diagnosis and management. Am J Cardiovasc Drugs. 2006;6(1):9-14.

8. Plemmons RM, Dooley DP, Longfield RN. Septic thrombophlebitis of the portal vein (pylephlebitis): diagnosis and management in the modern era. Clin Infect Dis 1995;21:1114-1120.

9. Choudhry AJ, Baghdadi YMK, Amr MA, Alzghari MJ, Jenkins DH, Zielinski MD. Pylephlebitis: a Review of 95 Cases. J Gastrointest Surg. 2016;20(3):656-61.

10. Garrett A, Carnish E, Das N, Slome M, Measley R. Once Universally Fatal: Pylephlebitis. Am J Med. 2014;127(7):595-7.

11. Kanellopoulou T, Alexopoulou A, Theodossiades G, Koskinas J, Archimandritis AJ. Pylephlebitis: An overview of non-cirrhotic cases and factors related to outcome. Scand J Infect Dis. 2010;42(11-12):804-11.

12. Plessier A, Darwish-Murad S, Hernandez-Guerra M, Consigny Y, Fabris F, Trebicka J, et al. Acute portal vein thrombosis unrelated to cirrhosis: A prospective multicenter follow-up study. Hepatology. 2010;51(1):210-8.

13. EASL Clinical Practice Guidelines: Vascular diseases of the liver. J Hepatol. 2016 Jan;64(1):179-202.

14. Altamirano J, Zapata L, Poblano M, Rodríguez A, Camargo L, Martínez B, et al. Acute pylephlebitis following gastrointestinal infection: an unrecognized cause of septic shock: South Med J. 2010;103(9):956-9.

15. Liu Y, Wang J, Jiang W. An increasing prominent disease of Klebsiella pneumoniae liver abscess: etiology, diagnosis, and treatment. Gastroenterol Res Pract. 2013;2013:1-12. 
16. Coyne C, Jain A. Pylephlebitis in a previously healthy emergency department patient with appendicitis. West J Emerg Med. 2013;14(5):428-30.

17. Naymagon L, Tremblay D, Schiano T, Mascarenhas J. The role of anticoagulation in pylephlebitis: a retrospective examination of characteristics and outcomes. J Thromb Thrombolysis. 2020;49(2):325-31.

18. Maruyama H, Ishibashi H, Takahashi M, Shimada T, Kamesaki H, Yokosuka O. Prediction of the therapeutic effects of anticoagulation for recent portal vein thrombosis: a novel approach with contrast-enhanced ultrasound. Abdom Imaging. 2012;37(3):431-8.

19. Kearon C, Akl EA, Comerota AJ, Prandoni P, Bounameaux H, Goldhaber SZ, et al. Antithrombotic Therapy for VTE Disease. Chest. 2012;141(2):e419S-e496S.

20. Lewis M. Bleeding Colonic Diverticula: J Clin Gastroenterol. 2008 ;42(10):1156-8.

21. Adnan MM, Gavin M, Eberhardt SC, McCarthy DM. Pylephlebitis: through these portals pass bad bugs. Dig Dis Sci. 2016 Oct;61(10):2807-11. 